\title{
An ultrasonic auditory stimulator
}

\author{
WILLIAM J. MUNDL and HELEN P. MALMO \\ Neuropsychology Laboratory, Department of Psychiatry, McGill University \\ Montreal, Quebec H3A IAI, Canada
}

\begin{abstract}
An auditory stimulator with a frequency range to $40 \mathrm{kHz}$ is described. The onset and cessation of the stimulation tone are carefully shaped in order to eliminate unwanted clicks. Rats have shown marked responses in electrical brain activity to ultrasonic stimulation.
\end{abstract}

Marked responses in electrical activity in the reticular formation of a rat have been observed while the animal was subjected to auditory stimulation, with the stimulation frequency ranging from the normal human auditory band to the ultrasonic region (Malmo \& Malmo, Note 1). Such stimulation should not be produced by simply switching a signal on or off, since this gives rise to two undesirable artifacts: (1) Frequencies lower than the stimulation frequency are created whenever a signal is abruptly switched, and (2) audible amplifier noise increases as the signal amplitude increases, thereby acting as a stimulus in the human audible range, although the controlled stimulation might be in the ultrasonic range.

To elaborate on the above, an auditory stimulus of a duration that is much greater than the period of the stimulation frequency can be considered a step function. Fourier analysis of a step function results in a frequency spectrum ranging from zero to the stimulation frequency. Higher frequencies are also produced, although these are of less importance in ultrasonic stimulation. Step function analysis is described by Craig (1964) and Hoelzler and Holzwarth (1957). That the ear performs spectral analysis and that it is extremely sensitive to frequency differences within the spectrum is described by Leshowitz (1978).

Accordingly, a signal burst must therefore be shaped in such a way that its envelope is devoid of any abrupt transitions. Figure 1 shows a burst of an $8-\mathrm{kHz}$ signal with gradual transition at the onset and cessation. There are no audible clicks, which would indicate the presence of harmonic frequencies.

Figure 2 shows the electrical activity in the reticular formation of an anesthetized rat. It was recorded with a bipolar electrode made of 63.5-micrometer-diam wire $(80 \% \mathrm{Pt}, 20 \% \mathrm{Ir})$. The neuronal signal was processed in two ways: One trace showed integrated multiple-unit activity (MUA) (Mundl, 1971), and the other trace showed the larger individual spikes (Mundl, 1969; Mundl $\&$ Malmo, Note 2). The two types of recording of MUA

This work was supported by Canadian Medical Research Council Grant MA 6438. We thank R. B. Malmo for assistance with surgery and MUA recording and for critical reading of the manuscript and Margaret Ross for typing the manuscript.

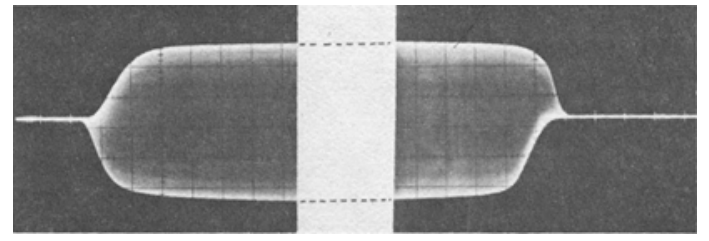

Figure 1. An 8-kHz tone burst with gradual onset and finish.
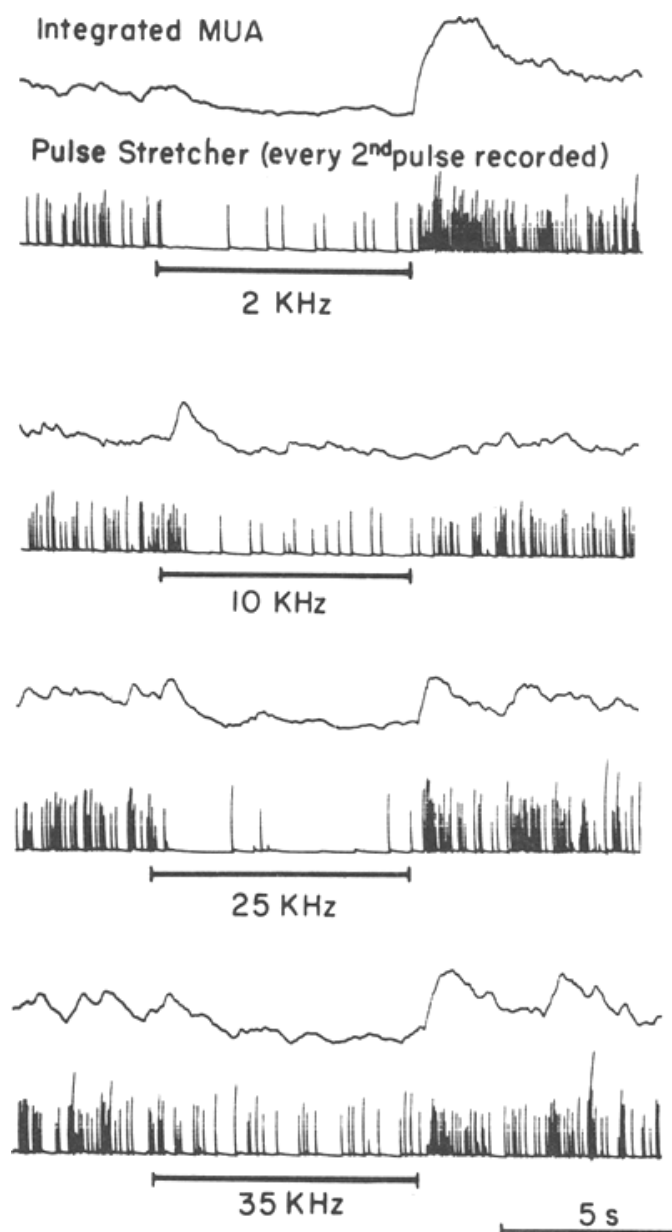

Figure 2. Neuronal response in the midbrain of a rat due to auditory stimulation. Inhibition and facilitation show up clearly. 


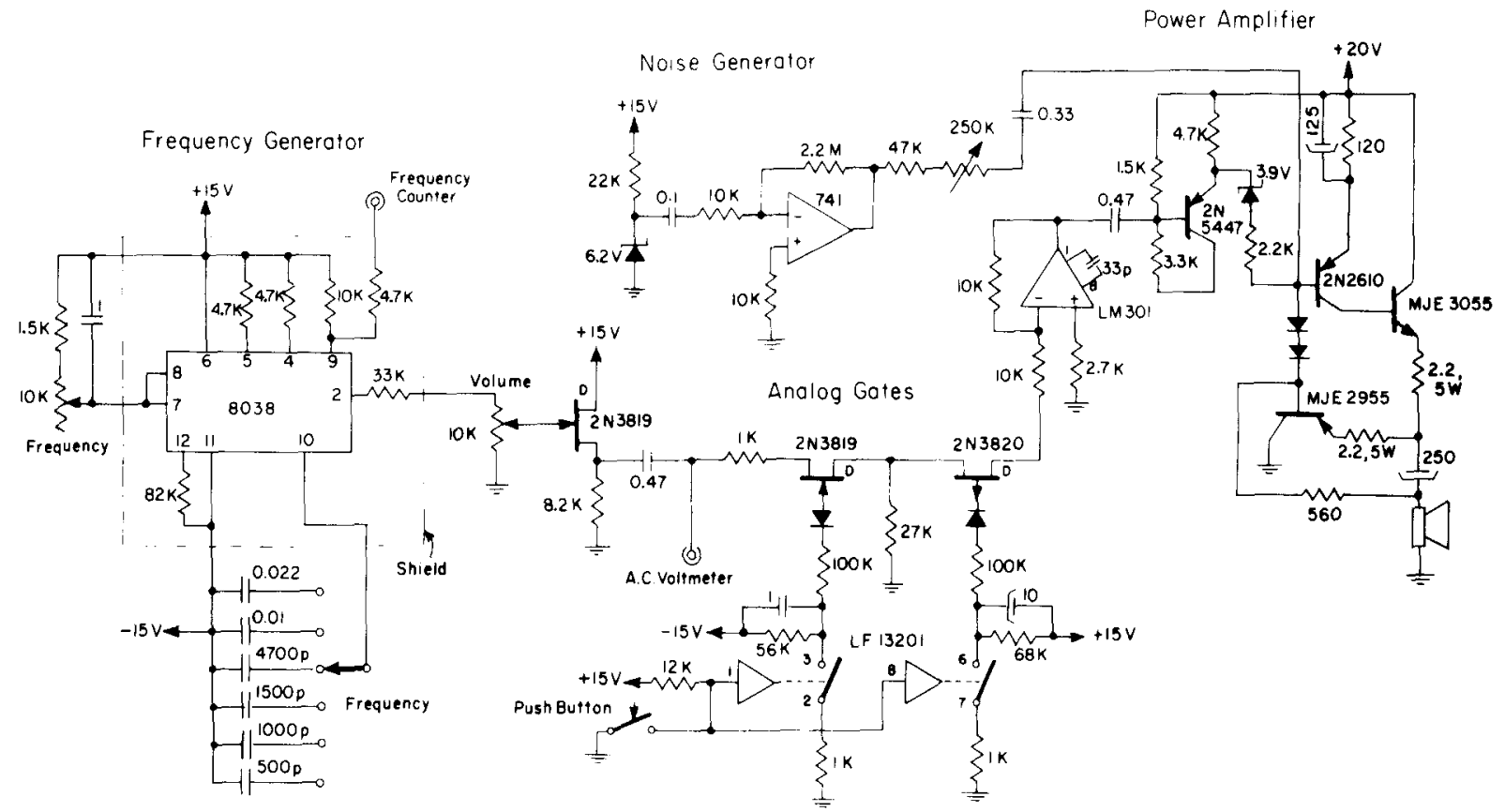

Figure 3. Circuit diagram. A tone is generated when the pushbutton switch is depressed. The Zener diode in the noise generator has been selected for a high level of inherent noise. An extemal frequency source may be used in place of the frequency generator.

complement each other, with the integrated MUA taking into account both the larger spikes and the more frequent smaller ones. Both inhibition and facilitation of neuronal activity can be seen in response to auditory stimulation. There is no consistent response at the onset or the cessation of the stimulation; if there were, it would indicate switching artifacts.

Bursts of ultrasonic signals with gradual rise and decay times were used by, for example, Moller (1969) and Rose, Greenwood, Goldberg, and Hind (1963). The shape of the envelope of these tone bursts, however, was not fully described.

\section{CIRCUIT}

As shown in Figure 3, the instrument contains its own signal generator (Intersil Type 8038 waveform generator). With different capacitors switched in, the generator produces frequencies from $500 \mathrm{~Hz}$ to $80 \mathrm{kHz}$. A frequency counter monitors frequency, and an ac meter indicates the signal amplitude after the volume control. A contact closure at the "Trigger" input activates two electronic switches (National Semiconductor Type 13201 analog switch), which, in turn, control the two fieldeffect transistor analog gates. These analog gates become gradually conducting, due to a corresponding slow voltage build up across the 1-microfarad and 10-microfarad capacitors. A similarly slow switching takes place at the end of the stimulus (i.e., when the pushbutton at the trigger input is released).
In order to eliminate audible clicks, it was found necessary to use two analog gates, one with a shorter and one with a longer time constant. One time constant is generated by the 1 -microfarad capacitor and $56-\mathrm{kohm}$ resistor, the other by the 10-microfarad capacitor and 68-kohm resistor.

The power amplifier drives a dome tweeter with a frequency response to $40 \mathrm{kHz}$ (Model T27, KEF Electronics, Ltd., Tovil Maidstone, England). Since the dome tweeter performs poorly at frequencies below $2 \mathrm{kHz}$, an ordinary loudspeaker may be substituted when lower frequencies are used. Also connected to the power amplifier is a noise source, which masks the noise produced when amplifying a strong signal.

The instrument is capable of producing a sound level of $95 \mathrm{~dB}$.

\section{REFERENCE NOTES}

1. Malmo, H. P., \& Malmo, R. B. Multiple-unit activity recorded longitudinally in rats from pubescence to old age. Manuscript submitted for publication, 1981.

2. Mundl, W. J., \& Malmo, H. P. Recording of unit activity on paper charts. Manuscript submitted for publication, 1980.

\section{REFERENCES}

Craig, E. J. Laplace and Fourier transforms for electrical engineers. New York: Holt, Rinehart, \& Winston, 1964.

Hoelzler, E., \& Holzwarth, H. Theorie und Technik der Pulsmodulation. Berlin: Springer-Verlag, 1957.

LEshowitz, B. Measurement of auditory stimulus. In E. C. Carterette \& M. P. Friedman (Eds.), Handbook of perception. New York: Academic Press, 1978. 
Mollen, A. R. Unit responses in the cochlear nucleus of the rat to pure tones. Acta Physiologica Scandinavica, 1969, 75, 530-541.

MUNDL, W. J. Stretching of analogue pulses. Electronic Engineering, 1969, 41, 215-217.

MUnDt, W. J. Preamplifier for recording of multiple-unit activity from moving animals. Physiology \& Behavior, 1971, 6, 617-618.

Rose, J. E., Greenwood, D. D., Goldberg, J. M., \& Hind, J. E.
Some discharge characteristics of single neurons in the inferior colliculus of the cat. 1 . Tonotopical organization, relation of spike counts to tone intensity, and firing patterns of single elements. Journal of Neurophysiology, 1963, 26, 294-320.

(Received for publication March 31, 1981; accepted April 18, 1981.) 